\title{
Charged reflecting shells supporting non-minimally coupled massless scalar field configurations
}

\author{
Shahar Hod ${ }^{1,2, a}$ \\ ${ }^{1}$ The Ruppin Academic Center, 40250 Emeq Hefer, Israel \\ 2 The Hadassah Academic College, 91010 Jerusalem, Israel
}

Received: 9 April 2020 / Accepted: 27 April 2020 / Published online: 12 May 2020

(C) The Author(s) 2020

\begin{abstract}
We study analytically the physical and mathematical properties of spatially regular massless scalar field configurations which are non-minimally coupled to the electromagnetic field of a spherically symmetric charged reflecting shell. In particular, the Klein-Gordon wave equation for the composed charged-reflecting-shell-nonminimallycoupled-linearized-massless-scalar-field system is solved analytically. Interestingly, we explicitly prove that the discrete resonance spectrum $\left\{R_{\mathrm{S}}(Q, \alpha, l ; n)\right\}_{n=1}^{n=\infty}$ of charged shell radii that can support the non-minimally coupled massless scalar fields can be expressed in a remarkably compact form in terms of the characteristic zeros of the Bessel function (here $Q, \alpha$, and $l$ are respectively the electric charge of the central supporting shell, the dimensionless non-minimal coupling parameter of the Maxwell-scalar theory, and the angular harmonic index of the supported scalar configuration).
\end{abstract}

\section{Introduction}

Black-hole spacetimes are characterized by the presence of event horizons, well defined boundaries on which matter and radiation fields are characterized by purely ingoing (absorbing) boundary conditions. This remarkable property of classical black-hole horizons has led Wheeler [1-3] to conjecture that static field configurations cannot be supported in the exterior regions of black-hole spacetimes with spatially regular horizons.

The absorbing boundary conditions, which characterize the behavior of matter fields on classical black-hole horizons, have played a key role in various no-hair theorems [4-11] that explicitly proved, in accord with Wheeler's conjecture [1-3], that spatially regular matter configurations made of minimally coupled scalar fields (or scalar fields with a non-

\footnotetext{
a e-mail: shaharhod@gmail.com (corresponding author)
}

minimal coupling to the Ricci curvature scalar) cannot be supported in black-hole spacetimes.

Intriguingly, recent studies [12-15] have revealed the fact that absorbing boundary conditions are actually not a necessary condition for a no-hair property of compact physical objects. In particular, the no-hair theorems presented in [1214] have explicitly proved that spherically symmetric horizonless compact stars with reflecting (rather than absorbing) boundary conditions cannot support spatially regular scalar field configurations.

Interestingly, it has recently been proved in the physically important works $[16,17]$ (see also [18]) that the no-hair conjecture can be violated in composed Einstein-Maxwellscalar theories in which the scalar fields are non-minimally coupled to the Maxwell electromagnetic tensor. In particular, it has been explicitly demonstrated in $[16,17]$ that spherically symmetric charged black holes can support massless scalar field configurations which are non-minimally coupled to the electromagnetic field of the central supporting charged black hole (See [19-21] for the interesting case of scalar fields which are non-minimally coupled to the Gauss-Bonnet invariant and supported by black holes and reflecting shells.).

In the present compact paper we shall explicitly prove that, similarly to the familiar case of charged black-hole spacetimes with absorbing boundary conditions, charged compact shells with reflecting boundary conditions can also support spatially regular massless scalar field configurations which are characterized by a non-minimal coupling to the electromagnetic field of the central supporting shell.

In particular, using analytical techniques (The case of massive non-minimally coupled scalar fields was studied numerically in the interesting work [22]. In the present paper we shall explicitly prove that the case of massless non-minimally coupled scalar fields can be studied analytically), we shall explicitly prove below that, for a spherically symmetric compact reflecting shell of electric charge $Q$, there exists an infinitely large discrete resonance spec- 
trum $\left\{R_{\mathrm{S}}(Q, \alpha ; n)\right\}_{n=1}^{n=\infty}$ of shell radii that can support nonminimally coupled massless scalar field configurations [here the physical parameter $\alpha$ is the dimensionless coupling parameter between the supported scalar field and the electromagnetic field of the central charged shell, see Eq. (3) below].

\section{Description of the system}

We shall analyze the physical and mathematical properties of massless scalar field configurations which are non-minimally coupled to the electromagnetic field of charged reflecting shells.

The spatial behavior of the non-minimally coupled scalar field configurations is determined by the differential equation (The scalar field equation (1) follows from the Lagrangian density $2 \nabla_{\alpha} \phi \nabla^{\alpha} \phi+f(\phi) \mathcal{I}[16,17]$. In addition, in order to facilitate a fully analytical treatment of the composed charged-shell-nonminimally-coupled-massless-scalar-field system, we shall work within the flat-space approximation, in which case the metric is described by the spherically symmetric line element $d s^{2}=-d t^{2}+d r^{2}+r^{2}\left(d \theta^{2}+\sin ^{2} \theta d \phi^{2}\right)$.) (We shall use natural units in which $\hbar=c=1$.) $[16,17]$

$\nabla^{\nu} \nabla_{\nu} \phi=\frac{1}{4} f_{, \phi} \mathcal{I}$,

where the source term $\mathcal{I}$, which governs the non-trivial coupling between the supported scalar field $\phi$ and the electromagnetic Maxwell tensor $F_{\mu \nu}$ of the central charged shell, is given by the simple expression (Note that $\mathcal{I}=Q^{2} / r^{4}$ in the weak-field regime.)

$\mathcal{I}=F_{\mu \nu} F^{\mu \nu}$.

In the weak-field regime, the scalar coupling function $f(\phi)$ is assumed to be characterized by the quadratic behavior [1618]

$f(\phi)=1+\alpha \phi^{2}$,

where the dimensionless physical parameter $\alpha$ (We shall henceforth assume that $\alpha>0$.) couples the scalar field to the electromagnetic field of the charged supporting shell (It is important to emphasize the physically interesting fact that, as nicely shown in [23], this class of models with a different coupling function $f(\phi)$ also allows for the existence of everywhere regular (solitonic) field configurations.).

In order to facilitate a fully analytical treatment of the composed charged-shell-nonminimally-coupled-masslessscalar-field system, we shall work within the flat-space approximation, in which case the scalar equation (1) can be written in the compact mathematical form $\frac{d}{d r}\left(r^{2} \frac{d R_{l m}}{d r}\right)-\left[l(l+1)-\frac{\alpha Q^{2}}{r^{2}}\right] R_{l m}=0$,

where $Q$ is the electric charge of the central supporting shell (Without loss of generality, we shall assume $Q>0$ for the electric charge of the compact shell.). The radial scalar eigenfunction $R_{l m}(r)$ is defined by the relation

$\phi(r, \theta, \phi)=\sum_{l m} R_{l m}(r) Y_{l m}(\theta) e^{i m \phi}$.

Here the integer parameters $\{l, m\}$ are the angular harmonic indices of the supported scalar field modes [note that the characteristic eigenvalue of the angular scalar eigenfunction $Y_{l m}(\theta)$ is given by the simple expression $\left.l(l+1)\right]$.

Below we shall consider spatially regular non-minimally coupled scalar field configurations whose radial profiles decay asymptotically $[16,17]$,

$\phi(r \rightarrow \infty) \rightarrow 0$.

In addition, we shall assume that the charged shell, which supports the non-minimally coupled scalar field configurations, is characterized by a reflecting surface of radius $R_{\mathrm{S}}$ with Dirichlet boundary conditions. This property of the central supporting shell dictates the inner reflecting boundary condition

$\phi\left(r=R_{\mathrm{S}}\right)=0$

for the composed charged-shell-nonminimally-coupledmassless-scalar-field configurations.

As we shall now prove, the radial differential equation (4) for the supported scalar field configurations, supplemented by the boundary conditions (6) and (7), determines an infinitely large discrete resonance spectrum of radii, $\left\{R_{\mathrm{S}}(Q, \alpha, l ; n)\right\}_{n=1}^{n=\infty}$, which, for given values $\{Q, \alpha, l\}$ of the physical parameters of the system, characterize the central reflecting shells that can support the nonminimally coupled static scalar field configurations. In particular, in the next section we shall explicitly demonstrate that the physical and mathematical properties of the composed charged-reflecting-shell-nonminimally-coupledmassless-scalar-field configurations can be studied analytically.

\section{The resonance spectrum of the composed charged-reflecting-shell-nonminimally-coupled- massless-scalar-field configurations}

In the present section we shall determine analytically the discrete resonance spectrum $\left\{R_{\mathrm{S}}(Q, \alpha, l ; n)\right\}_{n=1}^{n=\infty}$ which characterizes the charged reflecting shells that can support the spatially regular static configurations of the non-minimally 
coupled massless scalar fields. This to this, it proves useful to define the radial scalar eigenfunction

$\psi_{l m} \equiv r R_{l m}$,

in terms of which the radial equation (4) can be written in the form

$\frac{d^{2} \psi_{l}}{d r^{2}}-V_{l} \psi=0$

of a Schrödinger-like ordinary differential equation. Here $[16,17]$

$V_{l} \equiv V(r ; l, \alpha)=\frac{l(l+1)}{r^{2}}-\frac{\alpha Q^{2}}{r^{4}}$

is the effective radial potential of the composed chargedshell-scalar-field system (For brevity, we shall henceforth omit the angular harmonic parameters $l$ and $m$.).

Interestingly, as we shall now show, the Schrödinger-like radial equation (9), which determines the spatial behavior of the static non-minimally coupled scalar configurations, is amenable to an analytical treatment. In particular, the general mathematical solution of this radial differential equation can be expressed in terms of the Bessel function of the first kind (see Eq. 9.1 .53 of [24]):

$\psi(r)=A \cdot r^{\frac{1}{2}} J_{l+\frac{1}{2}}\left(\frac{\sqrt{\alpha} Q}{r}\right)+B \cdot r^{\frac{1}{2}} J_{-\left(l+\frac{1}{2}\right)}\left(\frac{\sqrt{\alpha} Q}{r}\right)$,

where $A$ and $B$ are normalization constants.

Using the characteristic small- $x$ behavior (see Eq. 9.1.10 of [24])

$J_{v}(x \rightarrow 0)=\frac{\left(\frac{1}{2} x\right)^{v}}{\Gamma(v+1)} \cdot\left[1+O\left(x^{2}\right)\right]$

of the Bessel functions, one finds that the asymptotic large- $r$ ( $\sqrt{\alpha} Q / r \rightarrow 0$ ) behavior of Eq. (11) is given by

$$
\begin{aligned}
\psi(r \rightarrow \infty)= & A\left[\Gamma\left(l+\frac{3}{2}\right)\right]^{-1} \cdot\left(\frac{\sqrt{\alpha} Q}{2}\right)^{l+\frac{1}{2}} r^{-l} \\
& +B\left[\Gamma\left(-l+\frac{1}{2}\right)\right]^{-1} \cdot\left(\frac{\sqrt{\alpha} Q}{2}\right)^{-\left(l+\frac{1}{2}\right)} r^{l+1} .
\end{aligned}
$$

Taking cognizance of the asymptotic boundary condition (6), one deduces the simple relation $B=0$. We therefore find that the spatially regular static configurations of the nonminimally coupled massless scalar fields are characterized by the radial functional behavior

$\psi(r)=A \cdot r^{\frac{1}{2}} J_{l+\frac{1}{2}}\left(\frac{\sqrt{\alpha} Q}{r}\right)$.

Taking cognizance of the inner boundary condition (7), which characterizes the behavior of the supported scalar fields on the surface of the central reflecting shell, one finds that the composed charged-reflecting-shell-nonminimallycoupled-massless-scalar-field system is characterized by the discrete resonance spectrum

$R_{\mathrm{S}}(Q, \alpha, l ; n)=\frac{\sqrt{\alpha} Q}{j_{l+\frac{1}{2}, n}} ; \quad n=1,2,3, \ldots$

of the supporting shell radii. Here $n=1,2,3, \ldots$ is the resonance parameter of the system and $j_{l+\frac{1}{2}, n}$ is the $n$th positive zero of the Bessel function $J_{l+\frac{1}{2}}(x)$. The real zeros of the Bessel function were studied by many authors, see e.g. $[24,25]$.

\section{Neumann boundary conditions}

In the previous section we have analyzed the physical properties of non-minimally coupled scalar field configurations which are supported by a charged reflecting shell with reflecting Dirichlet boundary conditions [see Eq. (7)]. It is also physically interesting to study the case of Neumann boundary conditions

$\frac{d \phi}{d r}=0 \quad$ for $\quad r=R_{\mathrm{S}}$

at the surface of the supporting shell.

Taking cognizance of Eqs. (5), (8), and (14), one can write the inner boundary condition (16) in the form

$\frac{d\left[r^{-\frac{1}{2}} J_{l+\frac{1}{2}}\left(\frac{\sqrt{\alpha} Q}{r}\right)\right]}{d r}=0$ for $r=R_{\mathrm{S}}$.

Using Eq. 9.1.31 of [24], one can express Eq. (17) in the form

$$
\begin{aligned}
J_{l+\frac{1}{2}} & \left(\frac{\sqrt{\alpha} Q}{R_{\mathrm{S}}}\right) \\
& +\frac{\sqrt{\alpha} Q}{R_{\mathrm{S}}}\left[J_{l-\frac{1}{2}}\left(\frac{\sqrt{\alpha} Q}{R_{\mathrm{S}}}\right)-J_{l+\frac{3}{2}}\left(\frac{\sqrt{\alpha} Q}{R_{\mathrm{S}}}\right)\right]=0 .
\end{aligned}
$$

As we shall now show explicitly, the rather cumbersome resonance equation (18) can be solved analytically in the regime

$\frac{\sqrt{\alpha} Q}{R_{\mathrm{S}}} \gg 1$

of small shell radii. In particular, using Eq. 9.2.1 of [24], one can write (18) in the remarkably compact form

$\cos \left(\frac{\sqrt{\alpha} Q}{R_{\mathrm{S}}}-\frac{1}{2} l \pi\right)+O\left[\left(\frac{\sqrt{\alpha} Q}{R_{\mathrm{S}}}\right)^{-1}\right]=0$.

From the resonance condition (20), one finds that the composed charged-reflecting-shell-nonminimally-coupledmassless-scalar-field system with reflecting Neumann bound- 
ary conditions is characterized by the resonance spectrum

$R_{\mathrm{S}}(Q, \alpha, l ; n)=\frac{\sqrt{\alpha} Q}{\left(n-\frac{1}{2}+\frac{1}{2} l\right) \pi} ; \quad n=1,2,3, \ldots$

in the regime (19) of small shell radii (Note that the regime (19) of small shell radii corresponds to large- $n$ values and/or lareg- $l$ values.).

\section{Summary and discussion}

Classical black holes with absorbing boundary conditions cannot support minimally coupled scalar field configurations [4-11]. Interestingly, this remarkable property is also shared by compact reflecting stars with repulsive (rather than absorbing) boundary conditions.

Intriguingly, the recently published works $[16,17]$ have revealed the physically important fact that charged blackhole spacetimes with regular event horizons can support massless scalar field configurations which are non-minimally coupled to the Maxwell tensor of the charged spacetime.

In the present compact paper we have explicitly proved that charged reflecting shells in flat spacetimes, like charged absorbing black holes, can support spatially regular configurations of massless scalar fields which are non-minimally coupled to the Maxwell tensor of the central compact shell. In particular, using analytical techniques, we have derived the remarkably compact dimensionless formula [see Eq. (15)] (The analytically derived resonance formula (22) is valid for the physical case of reflecting Dirichlet boundary conditions. See (21) for the case of reflecting Neumann boundary conditions.)

$\alpha\left(R_{\mathrm{s}}, Q, l ; n\right)=\left(\frac{R_{\mathrm{s}}}{Q}\right)^{2} \times j_{l+\frac{1}{2}, n}^{2} ; \quad n=1,2,3, \ldots$

for the discrete resonance spectrum that characterizes the physical coupling parameter $\alpha$ of the non-trivially coupled Maxwell-scalar theory (The physical parameter $\alpha$ couples the massless scalar field directly to the electromagnetic Maxwell tensor of the central charged sphere [see Eqs. (1), (2), and (3)].).

The analytically derived resonance spectrum (22) implies that, for given values $\left\{R_{\mathrm{S}}, Q\right\}$ of the physical parameters of the central supporting shell, the dimensionless coupling parameter $\alpha$ of the composed charged-shell-nonminimallycoupled-massless-scalar-field theory is an increasing function of the resonance parameter $n$. In particular, in the regime $n \gg l$ of large overtone numbers, one may use the asymptotic relation (see Eqs. 9.5.12 of [24]) $j_{l+\frac{1}{2}, n}=$ $\pi\left[n+\frac{1}{2} l+O\left(n^{-1}\right)\right]$ for the zeros of the Bessel function, which yields the asymptotic large- $n$ behavior

$\alpha(n \gg l)=\left(\frac{\pi R_{\mathrm{s}}}{Q}\right)^{2} \times\left(n+\frac{1}{2} l\right)^{2}$

of the resonance spectrum.

In addition, the resonance spectrum (22) implies that the dimensionless coupling parameter $\alpha$ of the composed charged-shell-nonminimally-coupled-massless-scalar-field configurations is an increasing function of the angular harmonic index $l$. In particular, in the regime $l \gg n$ of large angular harmonic indices, one may use the asymptotic relation (see Eq. 9.5.14 of [24]) $j_{l+\frac{1}{2}, n}=\left(l+\frac{1}{2}\right)\left[1+O\left(l^{-2 / 3}\right)\right]$ for the zeros of the Bessel function, which yields the asymptotic large- $l$ behavior (Note that (22), (23), and (24) are dimensionless expressions.)

$\alpha(l \gg n)=\left(\frac{R_{\mathrm{s}}}{Q}\right)^{2} \times l^{2}$

of the resonance spectrum.

Acknowledgements This research is supported by the Carmel Science Foundation. I would like to thank Yael Oren, Arbel M. Ongo, Ayelet B. Lata, and Alona B. Tea for helpful discussions.

Data Availability Statement This manuscript has no associated data or the data will not be deposited. [Authors' comment: I would like to emphasize that all relevant physical and mathematical calculations are explicitly presented in this paper.]

Open Access This article is licensed under a Creative Commons Attribution 4.0 International License, which permits use, sharing, adaptation, distribution and reproduction in any medium or format, as long as you give appropriate credit to the original author(s) and the source, provide a link to the Creative Commons licence, and indicate if changes were made. The images or other third party material in this article are included in the article's Creative Commons licence, unless indicated otherwise in a credit line to the material. If material is not included in the article's Creative Commons licence and your intended use is not permitted by statutory regulation or exceeds the permitted use, you will need to obtain permission directly from the copyright holder. To view a copy of this licence, visit http://creativecomm ons.org/licenses/by/4.0/.

Funded by SCOAP ${ }^{3}$.

\section{References}

1. R. Ruffini, J.A. Wheeler, Phys. Today 24, 30 (1971)

2. B. Carter, in Black Holes. Proceedings of 1972 Session of Ecole d'ete de Physique Theorique, edited by C. De Witt and B. S. De Witt (Gordon and Breach, New York, 1973)

3. J.D. Bekenstein, Phys. Today 33, 24 (1980)

4. J.D. Bekenstein, Phys. Rev. D 5, 1239 (1972)

5. C.A.R. Herdeiro, E. Radu, Int. J. Mod. Phys. D 24, 1542014 (2015)

6. S. Hod, Phys. Lett. B 713, 505 (2012)

7. S. Hod, Phys. Lett. B 718, 1489 (2013). [arXiv:1304.6474]

8. S. Hod, Phys. Rev. D 91, 044047 (2015). [arXiv:1504.00009]

9. A.E. Mayo, J.D. Bekenstein, Phys. Rev. D 54, 5059 (1996)

10. S. Hod, Phys. Lett. B 771, 521 (2017). arXiv: 1911.08371

11. S. Hod, Phys. Rev. D 96, 124037 (2017). arXiv:2002.05903 
12. S. Hod, Phys. Rev. D 94, 104073 (2016). arXiv: 1612.04823

13. S. Hod, Phys. Rev. D 96, 024019 (2017). arXiv: 1709.01933

14. S. Hod, Phys. Lett. B 773, 208 (2017)

15. S. Bhattacharjee, S. Sarkar, Phys. Rev. D 95, 084027 (2017)

16. C.A.R. Herdeiro, E. Radu, N. Sanchis-Gual, J.A. Font, Phys. Rev. Lett. 121, 101102 (2018)

17. P.G.S. Fernandes, C.A.R. Herdeiro, A.M. Pombo, E. Radu, N. Sanchis-Gual, Class. Quant. Grav. 36, 134002 (2019). arXiv: 1902.05079

18. S. Hod, Phys. Lett. B 798, 135025 (2019). arXiv:2002.01948

19. D.D. Doneva, S.S. Yazadjiev, Phys. Rev. Lett. 120, 131103 (2018)
20. H.O. Silva, J. Sakstein, L. Gualtieri, T.P. Sotiriou, E. Berti, Phys. Rev. Lett. 120, 131104 (2018)

21. Y. Peng, Eur. Phys. J. C 80, 202 (2020)

22. Y. Peng, Phys. Lett. B 804, 135372 (2020)

23. C.A.R. Herdeiro, J.M.S. Oliveira, E. Radu,. arXiv:1910.11021

24. M. Abramowitz, I.A. Stegun, Handbook of Mathematical Functions (Dover Publications, New York, 1970)

25. K.T. Tang, Mathematical Methods for Engineers and Scientists 3: Fourier Analysis, Partial Differential Equations and Variational Models (Springer, New York, 2006) 